\title{
Spin-liquid ground state in the frustrated kagome antiferromagnet $\mathrm{MgCu}_{3}(\mathrm{OH})_{6} \mathrm{Cl}_{2}$
}

\author{
E. Kermarrec, ${ }^{1}$ P. Mendels, ${ }^{1}$ F. Bert, ${ }^{1}$ R. H. Colman, ${ }^{2}$ A. S. Wills, ${ }^{2}$ P. Strobel, ${ }^{3}$ P. Bonville, ${ }^{4}$ A. Hillier,${ }^{5}$ and A. Amato ${ }^{6}$ \\ ${ }^{1}$ Laboratoire de Physique des Solides, Université Paris Sud, UMR CNRS 8502, F-91405 Orsay, France \\ ${ }^{2}$ University College London, Department of Chemistry, 20 Gordon Street, London, WC1H OAJ, United Kingdom \\ ${ }^{3}$ Institut Néel, Université Joseph Fourier, CNRS, F-38042 Grenoble, France \\ ${ }^{4}$ Service de Physique de l'État Condensé, CEA-CNRS, CE-Saclay, F-91191 Gif-Sur-Yvette, France \\ ${ }^{5}$ ISIS Facility, Rutherford Appleton Laboratory, Chilton, Didcot, Oxon OX11 OQX, United Kingdom \\ ${ }^{6}$ Laboratory for Muon Spin Spectroscopy, Paul Scherrer Institut, CH-5232 Villigen PSI, Switzerland
}

(Received 20 July 2011; published 6 September 2011)

\begin{abstract}
We report muon spin resonance experiments on $\mathrm{Mg}_{x} \mathrm{Cu}_{4-x}(\mathrm{OH})_{6} \mathrm{Cl}_{2}$ with $x \sim 1$, a material isostructural to Herbertsmithite exhibiting regular kagome planes of $S=\frac{1}{2}$ ions $\left(\mathrm{Cu}^{2+}\right)$, and therefore a candidate for a spin-liquid ground state. We evidence the absence of any magnetic ordering down to $20 \mathrm{mK}\left(\sim J / 10^{4}\right)$. We present a detailed investigation of the spin dynamics on well-characterized samples in zero and applied longitudinal fields, and a defect-based interpretation is proposed to explain the unconventional dynamics observed in the quantum spin-liquid phase.
\end{abstract}

DOI: 10.1103/PhysRevB.84.100401

PACS number(s): 75.10.Kt, 75.30.Hx, 76.75.+i

Quantum magnetism in frustrated networks has been acknowledged for a long time as the ideal playground to stabilize new quantum phases. Since the proposal of a resonating valence bond (RVB) state by Anderson, ${ }^{1}$ advances in theory have led to the emergence of a rich variety of spin-liquid phases, including algebraic and gapped spin liquids. ${ }^{2,3}$ On the experimental side, Herbertsmithite, $\mathrm{ZnCu}_{3}(\mathrm{OH})_{6} \mathrm{Cl}_{2}$, is among the best materials to explore such quantum states, as it combines the highly frustrated two-dimensional kagome lattice and quantum spins $\frac{1}{2}$ of $\mathrm{Cu}^{2+} .{ }^{4}$ No magnetic freezing has been detected down to $50 \mathrm{mK} \sim J / 4000$ (Refs. 5 and 6) and spin-spin correlations are found to be short ranged, as expected for a liquid state. ${ }^{7}$

Nevertheless, this material deviates from the "perfect" image since it has a sizable level of $\mathrm{Cu}-\mathrm{Zn}$ intersite mixing. The replacement of nonmagnetic $\mathrm{Zn}^{2+}$ ions at the interlayer position by magnetic $\mathrm{Cu}^{2+}$ causes almost free $S=\frac{1}{2}$ defects that correspond to $5 \%-10 \%$ of the total $\mathrm{Cu}$ content, as determined from various techniques (see Ref. 8 for a review). The exact amount of the complementary defect, where a nonmagnetic $\mathrm{Zn}^{2+}$ impurity induces a spin vacancy in the kagome plane, remains difficult to evaluate quantitatively, due to the similar x-ray scattering factors of $\mathrm{Cu}$ and $\mathrm{Zn}$, and the absence of a straightforward magnetic response. As a result, this value has been found to vary with samples and experimental techniques, from 1\% (Ref. 9) (from anomalous $\mathrm{x}$-ray scattering) to 5\% (Refs. 10 and 11) (from NMR).

Very recently, a different series, the "Mg-paratacamites," $\mathrm{Mg}_{x} \mathrm{Cu}_{4-x}(\mathrm{OH})_{6} \mathrm{Cl}_{2}$, which are isostructural to the $\mathrm{Zn}$-based paracatamite, has been successfully synthesized. The similar ionic radii of the diamagnetic $\mathrm{Mg}^{2+}$ and $\mathrm{Zn}^{2+}$ ions leads to a minimal difference in the crystal structure and hence in the exchange pathway, resulting in a comparable coupling of $J \sim 190 \mathrm{~K} .^{12-14}$ If $\mathrm{Cu} / \mathrm{Mg}$ mixing is still expected, the difference in their $\mathrm{x}$-ray scattering factors now enables a reliable x-ray structure analysis. Chu et al. ${ }^{15}$ succeeded in synthesizing materials for $x<0.75$, where they found a ferromagnetic component in macroscopic susceptibility under $T_{\mathrm{C}}=4 \mathrm{~K}$, attributed to a three-dimensional (3D) coupling via interlayer $\mathrm{Cu}^{2+}$, similar to the $\mathrm{Zn}$ case. A minimal $\mathrm{Mg}^{2+}$ substitution within the kagome planes $(\leqslant 3 \%)$ was determined through $\mathrm{x}$-ray diffraction. A different synthesis was recently reported which led to samples with $0.93 \leqslant x \leqslant$ $0.98 ;^{13}$ these correspond to the Herbertsmithite analogs and correspondingly to model quantum kagome antiferromagnets. A small ferromagnetic fraction at $T_{c} \simeq 4-5 \mathrm{~K}$ was tentatively ascribed to an impurity phase on the basis of susceptibility data.

In this Rapid Communication, we first report the muon spin resonance $(\mu \mathrm{SR})$ local probe investigation down to $20 \mathrm{mK}$ in the $\mathrm{Mg}_{x} \mathrm{Cu}_{4-x}(\mathrm{OH})_{6} \mathrm{Cl}_{2}$, "Mg-Herbertsmithites" with $x \sim 1$, which evidences a ground state with no sign of spin freezing, hence a spin-liquid character. We also study the sub-Kelvin unconventional spin dynamics of both paratacamites $(\mathrm{Mg}, \mathrm{Zn})$ characterized by an $x$-dependent plateau of $1 / T_{1}$. We argue that this relaxation is driven by interlayer $\mathrm{Cu}^{2+}$ ions.

The experiments were carried out on powder samples of $\mathrm{Mg}$ paratacamites close to the Herbertsmithite structure, $x=$ $0.84,0.92$, and 1.21 , synthesized following a hydrothermal route described elsewhere, ${ }^{13}$ as well as on the $\mathrm{Zn}$ Herbertsmithite $(x=1)$ sample from Ref. 16. The ratios of $\mathrm{Cu} / \mathrm{Mg}$ and $\mathrm{Cu} / \mathrm{Zn}$ were determined by inductively coupled plasma atomic emission spectroscopy (ICP-AES) (Table I). Refinements of x-ray diffraction data for the $\mathrm{Mg}$ compounds give separately the $\mathrm{Cu}(n)$ and $\mathrm{Mg}(p)$ occupancies of the interlayer and the kagome sites corresponding to the formula $\left(\mathrm{Cu}_{1-p} M_{p}\right)_{3}\left(M_{1-n} \mathrm{Cu}_{n}\right)(\mathrm{OH})_{6} \mathrm{Cl}_{2}(M=\mathrm{Mg}, \mathrm{Zn})$. The $M$ total amount is $x=3 p-n+1$ (Table I). A visible reduction in the quality of the fit, and a corresponding increase in the goodness-of-fit statistic ( $\chi^{2}=1.37-1.42$ for $x=0.92$ ), was found when attempting to fix $p=0$ as reported in Ref. 9, evidencing the ability of $\mathrm{x}$-ray diffraction to determine the metal site occupancies of the kagome and triangular sites. ${ }^{13}$ $\mu$ SR experiments were performed at the ISIS and PSI facilities in zero and longitudinal applied field configurations down to $20 \mathrm{mK}$. The dc magnetic susceptibility was also measured on a Quantum Design superconducting quantum interference device (SQUID) magnetometer in the $1.8-300 \mathrm{~K} T$ range, and 
TABLE I. Chemical composition determined through ICP, x-ray refinements, and saturated magnetization for $\left(\mathrm{Cu}_{1-p} M_{p}\right)_{3}\left(M_{1-n} \mathrm{Cu}_{n}\right)(\mathrm{OH})_{6} \mathrm{Cl}_{2}$, where $M=\mathrm{Mg}, \mathrm{Zn}$. Each site occupancy is constrained to unity.

\begin{tabular}{lcccc}
\hline \hline Element $M$ & $\mathrm{Mg}$ & $\mathrm{Mg}$ & $\mathrm{Zn}$ & $\mathrm{Mg}$ \\
\hline ICP & $x=0.84(1)$ & $x=0.92(1)$ & $x=1.00(7)$ & $x=1.25(3)$ \\
X-ray & $x=0.83^{\mathrm{a}}$ & $x=0.91^{\mathrm{a}}$ & - & $x=1.21(4)$ \\
$p$ & $0.041(1)$ & $0.063(1)$ & - & $0.12(1)$ \\
$n$ & $0.287(4)$ & $0.266(3)$ & & $0.15(1)$ \\
Magnetization & - & $0.266(4)$ & $0.217(5)$ & $0.186(4)$ \\
$n$ & - & & \\
\hline \hline
\end{tabular}

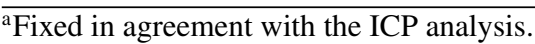

magnetization data were taken at $1.75 \mathrm{~K}$ in fields up to $14 \mathrm{~T}$ with a cryogenic vibrating sample magnetometer.

In order to reveal the magnetic response of the interplane defects, we measured the magnetization of the $\mathrm{Mg}(x=0.92$ and $x=1.21)$ and $\mathrm{Zn}(x=1)$ samples at low $T=1.75 \mathrm{~K}$ and up to $14 \mathrm{~T}$. Following the analysis in Refs. 13 and 16, we divide the total magnetization into two contributions, a Brillouin-like component $M_{\text {def }}$ arising from the weakly coupled interlayer $\mathrm{Cu}^{2+}$ and a linear term arising from the strongly coupled $\mathrm{Cu}^{2+}$ of the kagome planes (Fig. 1). By subtraction of the latter, we have access to the saturated magnetization $n M_{\text {sat }}$ of the interlayer $\mathrm{Cu}^{2+} .{ }^{17}$ Taking $g=2.2$ from Ref. 18 , this analysis provides an independent determination of $n$, which agrees well with the $\mathrm{X}$-ray refinements for the $\mathrm{Mg}$ samples and provides a value for the $\mathrm{Zn}$ sample otherwise inaccessible by structural studies (Table I). These results confirm quantitatively the existence of the weakly coupled $S=\frac{1}{2}$ interplane defects in the $\mathrm{Mg}$ and $\mathrm{Zn}$ paratacamites with $x \sim 1$.

We now turn to the local $\mu \mathrm{SR}$ investigations. $\mu \mathrm{SR}$ is very sensitive to any small magnetic field (down to $\sim 0.1 \mathrm{G}$ ) and is therefore a powerful tool to detect any frozen moment. The muon is implanted inside the volume and, as a positive charged

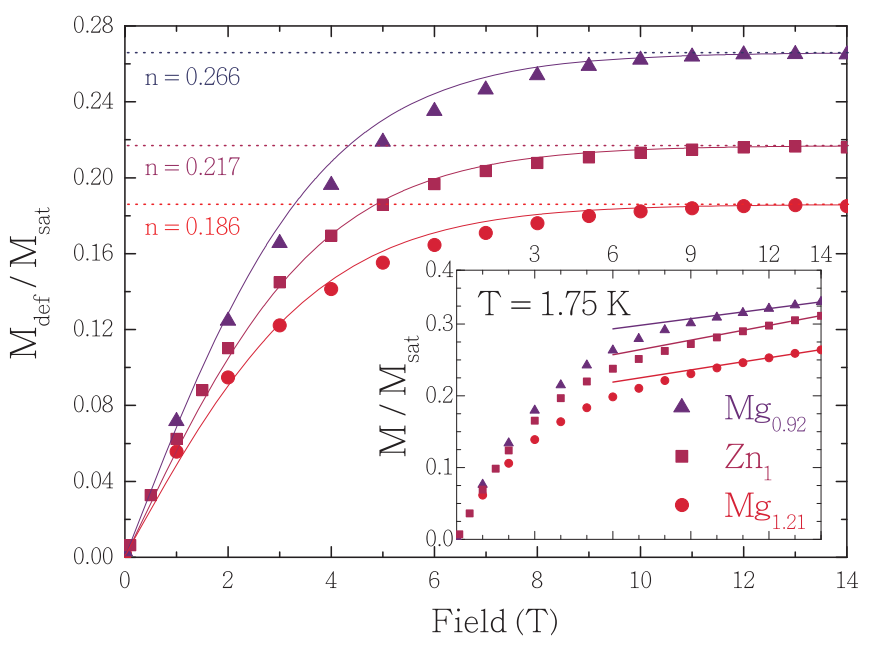

FIG. 1. (Color online) Interlayer $\mathrm{Cu}^{2+}$ contribution $M_{\text {def }}$ extracted from magnetization measurements and normalized by $M_{\text {sat }}=$ $N_{A} g \mu_{B} S=6143$ emu. The saturation value gives $n$, the amount of interlayer $\mathrm{Cu}^{2+}$ per formula unit. The lines reproduce a Brillouin fit with an interacting scale of $\theta=0.8 \mathrm{~K}$. Inset: Normalized magnetization at $T=1.75 \mathrm{~K}$ up to $14 \mathrm{~T}$. Lines are fits for the linear component. particle, will stop in the vicinity of a negative environment, i.e., either near $\mathrm{OH}^{-}$or $\mathrm{Cl}^{-5}$. The polarizations from 0 (ZF) to $2500 \mathrm{G}$ longitudinal applied field (LF) are reported in Fig. 2. Our experiments demonstrate a similar magnetic behavior of the $\mathrm{Mg}$ and $\mathrm{Zn}$ compounds, as expected due to their crystallographic similarities. Following the former work of Ref. 5, the ZF polarization is fitted on a high statistics run at $50 \mathrm{mK}$ by $P(t)=P_{\text {nucl }}(t) e^{-(\lambda t)^{\beta}} . P_{\text {nucl }}(t)$ depends only on static fields from surrounding nuclei (see Ref. 17), and $\lambda$ stands

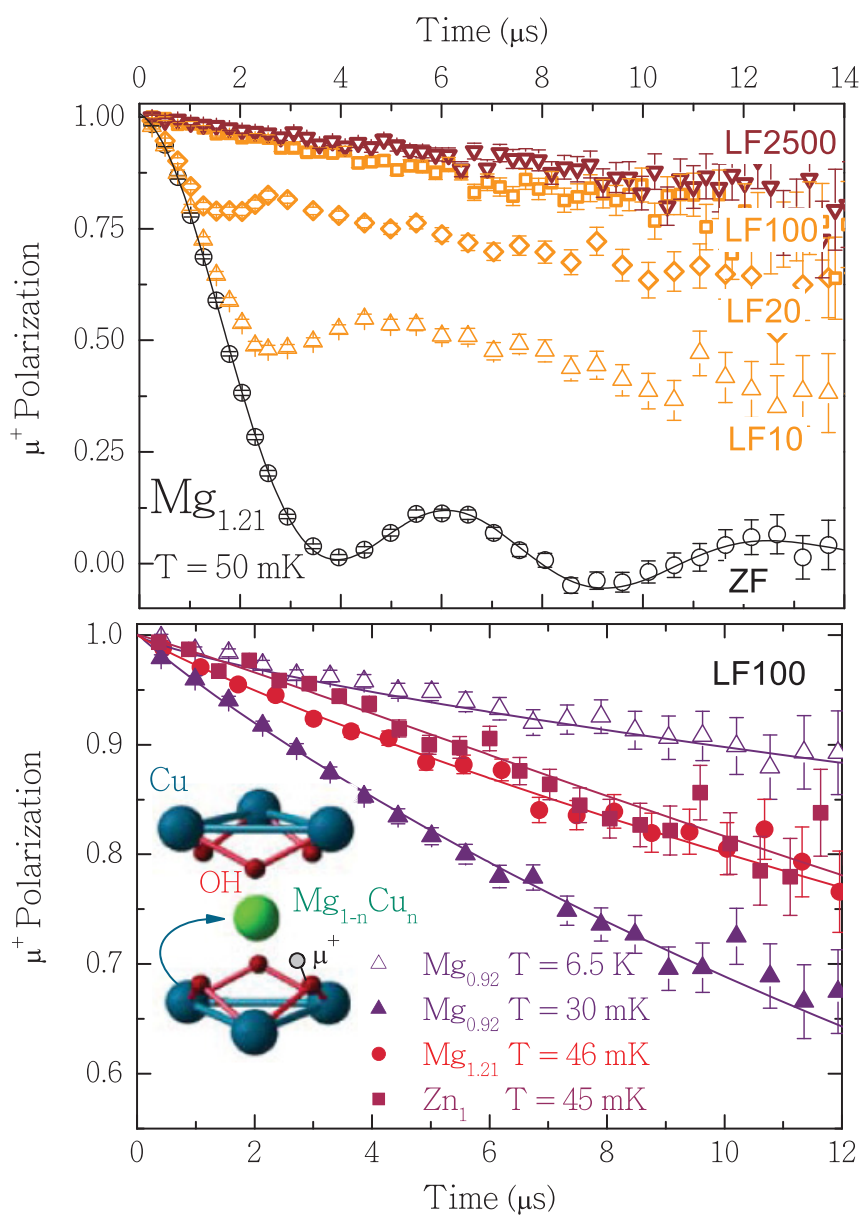

FIG. 2. (Color online) Upper panel: Polarization in zero field (ZF, black circles) and under longitudinal applied fields (LF) from 10 to $2500 \mathrm{G}$. The black line is a fit (see the text). Bottom panel: The relaxation is faster at low temperatures and when $n$ increases. Lines are stretched exponential fits. 


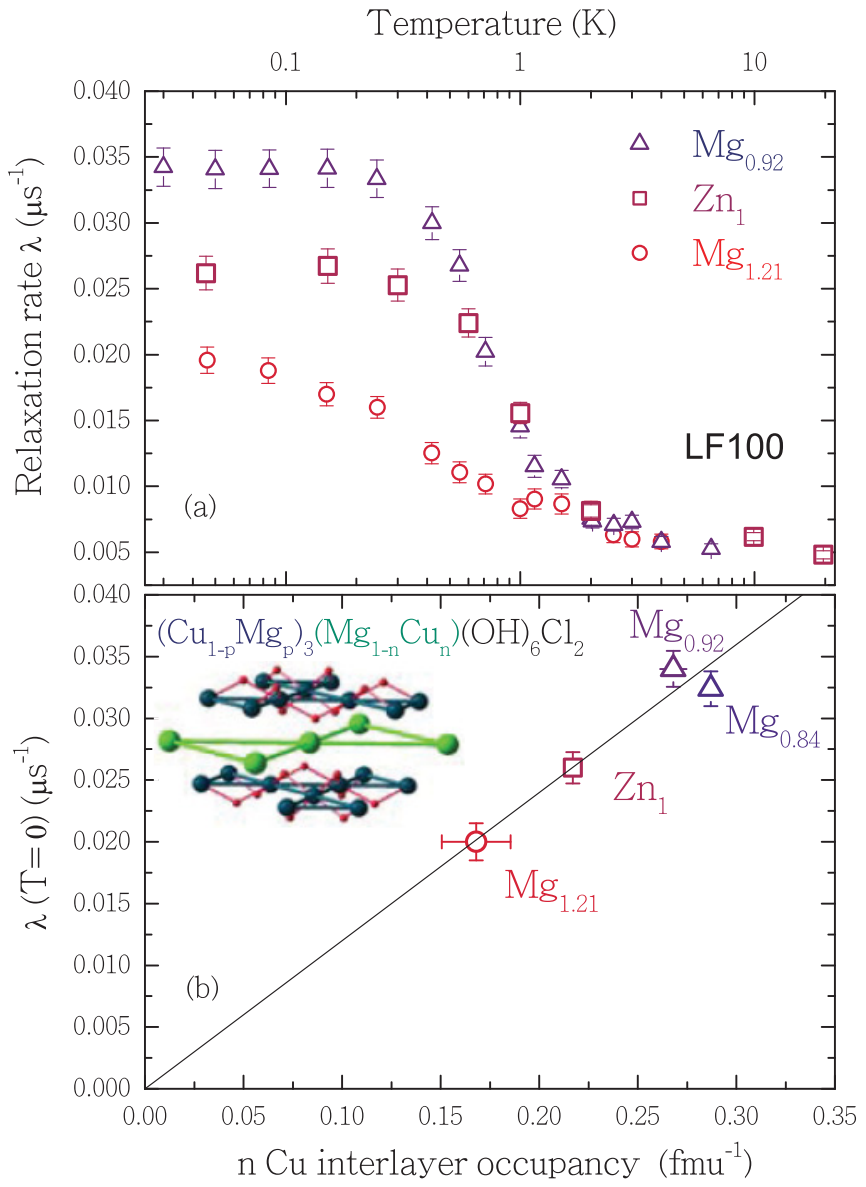

FIG. 3. (Color online) (a) $T$ dependence of relaxation rates $\lambda$ for different samples. (b) Plot of the plateau value of $\lambda=1 / T_{1}$ vs $n$. The black line is a linear fit. The error bars on $n$ account for the discrepancy between values derived from $M(H)$ and x-ray characterization.

for a small dynamical relaxation. The well-defined oscillation of $P_{\text {nucl }}(t)$ is due to the formation of a $\mu-\mathrm{O}-\mathrm{H}$ complex, ${ }^{19}$ and from the field experienced by the muon, $H_{\mu-\mathrm{OH}}=$ $7.8 \mathrm{G}$, one estimates a distance of $1.5 \AA$ to hydrogen. ${ }^{20}$ The $\mathrm{ZF}$ polarization is found to be largely unchanging in the extended $T$ range $0.05-20 \mathrm{~K}$, except for a slight variation of $\lambda$. Therefore, we conclude that there is no magnetic ordering of the electronic spins down to $T=20 \mathrm{mK}\left(\sim J / 10^{4}\right)$ for $x \sim 1$, as in the $\mathrm{Zn}$ counterpart.

The spin dynamics is revealed by experiments under longitudinal fields. From 10 to $100 \mathrm{G}$, the static relaxation of nuclear origin is progressively decoupled, as expected (Fig. 2). Above $100 \mathrm{G}\left(>10 H_{\mu-\mathrm{OH}}\right), P_{\text {nucl }}(t)=1$ and a single stretched exponential fit of $P(t)$ yields the relaxation rate $\lambda=1 / T_{1}^{\mu}$, which is of electronic origin. $\lambda=1 / T_{1}^{\mu}$ is linked to the spin autocorrelation function by $1 / T_{1}^{\mu} \sim \int_{0}^{+\infty}\langle\mathbf{S}(t) \mathbf{S}(0)\rangle \cos \left(\gamma_{\mu} H_{\mathrm{LF}} t\right) d t$, where $H_{\mathrm{LF}}$ is the longitudinal applied magnetic field. Starting from a high- $T$ value $\sim 0.005 \mu \mathrm{s}^{-1}, \lambda$ increases upon cooling below $1 \mathrm{~K}$ and saturates at lower temperature at a value which differs from sample to sample (Fig. 3). This increase of $\lambda$ indicates a dynamical slowing down. We notice that the shape of the relaxation at low $T$ slightly changes between $\mathrm{Mg}$ $(\beta=0.9)$ and $\mathrm{Zn}(\beta=1.1)$ compounds.
We now argue that the muon is dominantly coupled to the interlayer $\mathrm{Cu}^{2+}$ moments from the following experimental observations: (i) The low- $T$ muon shift $K^{\mu}$ was shown to track the intersite defect susceptibility. ${ }^{21}$ This is in fair agreement with a recent ${ }^{2} \mathrm{D}$ NMR study ${ }^{22}$ but contrasts with NMR results on ${ }^{17} \mathrm{O}$, which is strongly coupled to the planes. (ii) From Fig. 3(b) and inspection of Table I, $\lambda$ is found to increase linearly with $n$, the concentration of intersite defects, but is anticorrelated with the level of in-plane defects $p$. Since the distance between $\mathrm{Zn}$ sites is large $(d=6.12 \AA)$, the linearity in $n$ can be easily explained by an "all or nothing" model: Some muons $(n)$ sit next to an interlayer $\mathrm{Cu}^{2+}$ defect with a relaxation rate $\lambda_{1}$, whereas the others $(1-n)$ stay far from a defect with a relaxation rate $\lambda_{2} \rightarrow 0$. Under the valid condition $\lambda_{1,2} t \ll 1$, the total polarization becomes $P(t)=$ $n \exp \left(-\lambda_{1} t\right)+(1-n) \exp \left(-\lambda_{2} t\right) \sim \exp \left(-n \lambda_{1} t\right)$, which explains the linear $n$ dependence of $\lambda$. (iii) From the scaling of $K^{\mu}$ vs $\chi^{\text {bulk }}$, one can extract the coupling constant $A_{\mu}=$ $0.08 \mathrm{~T} / \mu_{B},{ }^{21}$ consistent with a dipolar interaction. From ${ }^{17} \mathrm{O}$ NMR $T_{1}^{17}$, the contribution of the kagome planes $\mathrm{Cu}^{2+}$ to the muon relaxation can then be estimated using $1 / T_{1}^{\mu}=$ $1 / T_{1}^{17}\left(\gamma_{\mu} A_{\mu} / \gamma_{\mathrm{n}}^{17} A_{\mathrm{hf}}^{17}\right)^{2} \sim 3 \times 10^{-5} \mu \mathrm{s}^{-1}$. This leads at high $T$ to a value 150 times smaller than the measured one, which therefore requires a different source of relaxation. (iv) Finally, in the high- $T$ Moriya paramagnetic limit, ${ }^{23} 1 / T_{1}^{\mu}$ is given by $1 / T_{1}^{\mu}=2 \gamma_{\mathrm{n}}^{2} H_{\mu}^{2} n / v$ with $v=\sqrt{4 J^{\prime 2} z S(S+1) / 3 \pi \hbar^{2}}$ and $H_{\mu}=g A_{\mu} \sqrt{S(S+1) / 3}=880 \mathrm{G}$. From the high- $T$ constant value $1 / T_{1}^{\mu} \sim 5 \times 10^{-3} \mu \mathrm{s}^{-1}$, an average value $n \sim 0.22$ and a number of nearest neighbors $z=6$, one obtains the coupling between an interlayer $\mathrm{Cu}^{2+}$ and a kagome $\mathrm{Cu}^{2+} J^{\prime} \sim 3 \mathrm{~K}$, in rough agreement with the temperature of the magnetic ordering $T_{\mathrm{C}}=6 \mathrm{~K}$ in the clinoatacamite, $\mathrm{Cu}_{2}(\mathrm{OH})_{3} \mathrm{Cl}$, parent compound. ${ }^{24-26}$

In this context, the slowing down below $1 \mathrm{~K}$ of intersite defects can be attributed either to the strengthening of the correlations with the two nearby kagome planes or to a coupling between two intersite defects mediated by the kagome plane to which they are coupled. The energy scale of $1 \mathrm{~K}$ for the interaction between defects was also evidenced in magnetization measurements. ${ }^{16}$

We now discuss the $T \rightarrow 0$ value of the relaxation rate. In order to reveal the dynamics of the correlated regime under $1 \mathrm{~K}$, we probe the excitation spectrum $\tilde{\mathcal{S}}(\omega)$ in the low-energy range by applying a longitudinal field $H_{\mathrm{LF}}=\omega / \gamma_{\mu}, H_{\mathrm{LF}}<0.25 \mathrm{~T}$. Two scenarios can be considered based on different correlation functions $\mathcal{S}(t)=\langle\mathbf{S}(t) \mathbf{S}(0)\rangle$ for the interplane spins.

(1) Exponential correlation function $\mathcal{S}(t)=e^{-v t}$ combined with field-induced polarization of the interlayer defects: Such a correlation leads to the usual Lorentzian spectral density $\tilde{\mathcal{S}}(\omega)$ and

$$
\lambda_{1}=\frac{2 \gamma_{\mu}^{2} H_{\text {fluct }}^{2} v}{v^{2}+\gamma_{\mu}^{2} H_{\mathrm{LF}}^{2}},
$$

where $H_{\text {fluct }}$ is the fluctuating component of the field at the muon site perpendicular to its initial polarization, $v$ is the fluctuation frequency, and $\gamma_{\mu}=851.6 \mathrm{Mrad} / \mathrm{s} / \mathrm{T}$ is the muon gyromagnetic factor. However, not only is the expected $H_{\mathrm{LF}}^{2}$ dependence of $\lambda$ not convincing (Fig. 4), but a forced fit results in an unphysical $H_{\text {fluct }} \sim 20 \mathrm{G}$, while the lowest possible 


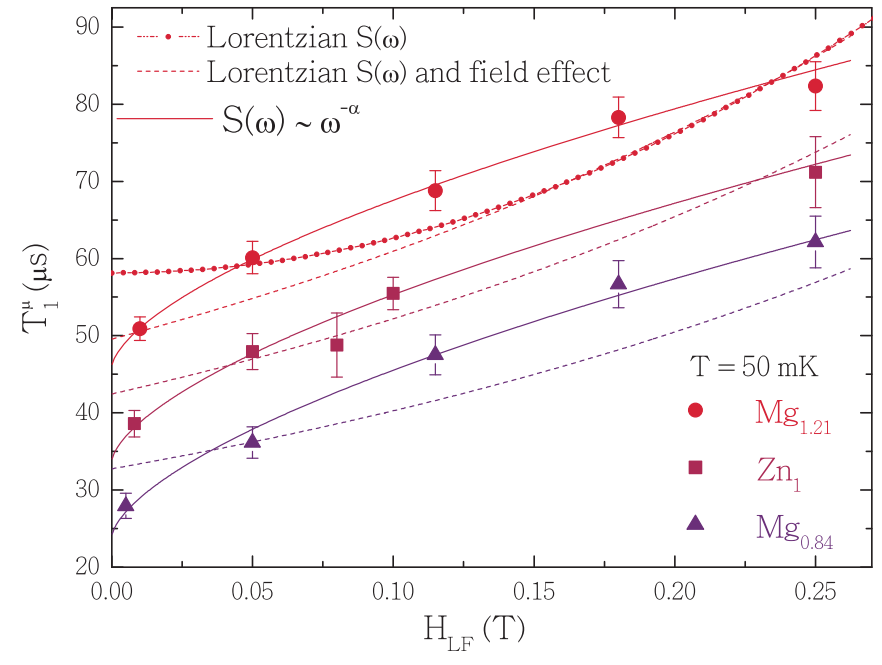

FIG. 4. (Color online) The $T_{1}$ dependence with external field $H_{\mathrm{LF}}$ underlines the unconventional spin dynamics at $T=50 \mathrm{mK}$. The lines refer to different models (see the text).

dipolar field value is $200 \mathrm{G}$, which corresponds to a maximal distance of the $\mu^{+}$to intersite $\mathrm{Cu}^{2+}$. We therefore propose that the fluctuating field is reduced when the interlayer $S=\frac{1}{2}$ defect starts to be polarized in the external applied field $H_{\mathrm{LF}}$. In a mean-field Brillouin approach, the fluctuating moment is $m^{\text {fluct }}=\mu_{B}\left(1-\tanh \left[g \mu_{B} S H_{\mathrm{LF}} / k_{B}(T+\theta)\right]\right)$, where $\theta$ is introduced to account for interactions. The reduced value of $H_{\text {fluct }}=m^{\text {fluct }} H_{\mu} / \mu_{B}$ can be injected in Eq. (1) and leads to only two shared fit parameters to account for the $T_{1}^{\mu}\left(H_{\mathrm{LF}}\right)$ variation for all $x$ samples. Although the obtained fits are not perfect, this approach yields $v=100 \pm 20 \mathrm{GHz}$, a typical frequency of fluctuations in a paramagnetic regime, and $\theta=$ $0.7 \pm 0.2 \mathrm{~K}$, consistent with both magnetization measurements and the $1 \mathrm{~K}$ temperature scale where the slowing down occurs.

(2) Power-law correlation function $\mathcal{S}(t)=(1 / t)^{1-\alpha}$ : The corresponding spectral density $\tilde{\mathcal{S}}(\omega)$ yields a power-law relation $T_{1}^{\mu} \propto \omega^{\alpha}$. This more exotic approach is at play $(\alpha=0.35)$ in the spin-liquid case of the $S=\frac{1}{2}$ antiferromagnetic chain, ${ }^{27}$ where a spinon continuum of excitations is well established. Such a spectral density ( $\alpha=1$ ) was also invoked for the spin liquid pyrochlore $\mathrm{TbTi}_{2} \mathrm{O}_{7}{ }^{28,29}$ A perfect fit of our data can be found with $1 / \lambda_{1}=T_{1}^{0}(x)+A \omega^{0.63}$, which would point to an exotic relaxation channel for the intersite defect. Using the scaling of $\chi^{\prime \prime} T^{\alpha} \sim(T / \omega)^{\alpha} \tanh (\omega / \beta T)$ from neutron experiments, ${ }^{30}$ one gets $1 / T_{1} \sim k_{B} T \chi^{\prime \prime} / \omega \sim \omega^{-\alpha}$ by means of the fluctuation dissipation theorem. The reported value $\alpha=0.66$ (Ref. 30) agrees well with that deduced from our data. This points to a common origin and, following Ref. 31, one could invoke a distribution of couplings between intersite defects as a source of the power law. Another possibility could be that the kagome dynamics drives that of the intersite defects. The inconsistency between the $T$ plateau of the relaxation rate measured at low fields and the $T^{-0.7}$ dependence of $T_{1}^{17}$ at $7 \mathrm{~T}$ (Ref. 10) would then require that the field impacts on the kagome plane dynamics.

In conclusion, the spin-liquid behavior of the kagome antiferromagnet $\mathrm{Mg}_{x} \mathrm{Cu}_{4-x}(\mathrm{OH})_{6} \mathrm{Cl}_{2}$ for $x>0.84$ is clearly established by our $\mu \mathrm{SR}$ experiments. Our data show that the measured interlayer site dynamics differs from those of the kagome plane at $7 \mathrm{~T}$. This calls for a careful inspection of probes which integrate both responses and/or the field impact on relaxation. Whether the exotic character of the $\mu^{+}$relaxation might also relate to the spin-liquid behavior of the kagome lattice is still a matter of speculation. The ability to refine the structure for "Mg-Herbertsmithite" in a reliable manner opens the possibility to control the level of defects and to discriminate between the various sources of dynamics at low $T$. Our results open routes for future investigations of the kagome spin-liquid ground state in well-controlled materials.

This research project has been supported by the European Commission under the 6th Framework Programme Contract No. RII3-CT-2003-505925 and Grant No. ANR-09-JCJC0093-01. We thank C. Baines for assistance at PSI and D. Dragoe for the ICP analysis.
${ }^{1}$ P. W. Anderson, Mater. Res. Bull. 8, 153 (1973).

${ }^{2}$ L. Balents, Nature (London) 464, 199 (2010).

${ }^{3}$ C. Lacroix, P. Mendels, and F. Mila, Introduction to Frustrated Magnetism (Springer, Berlin, 2010), p. 164.

${ }^{4}$ M. P. Shores, E. A. Nytko, B. M. Bartlett, and D. G. Nocera, J. Am. Chem. Soc. 127, 13462 (2005).

${ }^{5}$ P. Mendels, F. Bert, M. A. de Vries, A. Olariu, A. Harrison, F. Duc, J.-C. Trombe, J. S. Lord, A. Amato, and C. Baines, Phys. Rev. Lett. 98, 077204 (2007).

${ }^{6}$ J. S. Helton, K. Matan, M. P. Shores, E. A. Nytko, B. M. Bartlett, Y. Yoshida, Y. Takano, A. Suslov, Y. Qiu, J.-H. Chung, D. G. Nocera, and Y. S. Lee, Phys. Rev. Lett. 98, 107204 (2007).

${ }^{7}$ M. A. de Vries, J. R. Stewart, P. P. Deen, J. O. Piatek, G. J. Nilsen, H. M. Rønnow, and A. Harrison, Phys. Rev. Lett. 103, 237201 (2009).

${ }^{8}$ P. Mendels and F. Bert, J. Phys. Soc. Jpn. 79, 011001 (2010).
${ }^{9}$ D. E. Freedman, T. H. Han, A. Prodi, P. Müller, Q.-Z. Huang, Y.-S Chen, S. M. Webb, Y. S. Lee, T. M. McQueen, and D. G. Nocera, J. Am. Chem. Soc. 132, 16185 (2010).

${ }^{10}$ A. Olariu, P. Mendels, F. Bert, F. Duc, J.-C. Trombe, M. A. de Vries, and A. Harrison, Phys. Rev. Lett. 100, 087202 (2008).

${ }^{11} \mathrm{P}$. Mendels and F. Bert, e-print arXiv:1107.3038.

${ }^{12}$ M. Rigol and R. R. P. Singh, Phys. Rev. Lett. 98, 207204 (2007).

${ }^{13}$ R. H. Colman, A. Sinclair, and A. S. Wills, Chem. Mater. 23, 1811 (2011).

${ }^{14}$ G. Misguich and P. Sindzingre, Eur. Phys. J. B 59, 305 (2007).

${ }^{15}$ S. Chu, T. M. McQueen, R. Chisnell, D. E. Freedman, P. Müller, Y. S. Lee, and D. G. Nocera, J. Am. Chem. Soc. 132, 5570 (2010).

${ }^{16}$ F. Bert, S. Nakamae, F. Ladieu, D. L'Hôte, P. Bonville, F. Duc, J.-C. Trombe, and P. Mendels, Phys. Rev. B 76, 132411 (2007).

${ }^{17}$ See Supplemental Material at http://link.aps.org/supplemental/ 10.1103/PhysRevB.84.100401 for detailed expressions. 
${ }^{18}$ A. Zorko, S. Nellutla, J. van Tol, L. C. Brunel, F. Bert, F. Duc, J.-C. Trombe, M. A. de Vries, A. Harrison, and P. Mendels, Phys. Rev. Lett. 101, 026405 (2008).

${ }^{19}$ J. S. Lord, S. P. Cottrell, and W. G. Williams, Physica B 289, 495 (2000).

${ }^{20}$ J. H. Brewer, S. R. Kreitzman, D. R. Noakes, E. J. Ansaldo, D. R. Harshman, and R. Keitel, Phys. Rev. B 33, 7813 (1986).

${ }^{21}$ O. Ofer, A. Keren, J. H. Brewer, T. H. Han, and Y. S. Lee, J. Phys. Condens. Matter 23, 164207 (2011); P. Mendels and F. Bert (unpublished).

${ }^{22}$ T. Imai, M. Fu, T. H. Han, and Y. S. Lee, Phys. Rev. B 84, 020411(R) (2011).

${ }^{23}$ T. Moriya, Prog. Theor. Phys. 16, 23 (1956).

${ }^{24}$ A. S. Wills and J.-Y. Henry, J. Phys. Condens. Matter 20, 472206 (2008).

${ }^{25}$ A. S. Wills, T. G. Perring, S. Raymond, B. Fåk, J.-H. Henry, and M. Telling, J. Phys.: Conf. Ser. 145, 012056 (2009).
${ }^{26}$ X. G. Zheng, T. Kawae, Y. Kashitani, C. S. Li, N. Tateiwa, K. Takeda, H. Yamada, C. N. Xu, and Y. Ren, Phys. Rev. B 71, 052409 (2005).

${ }^{27}$ F. L. Pratt, S. J. Blundell, T. Lancaster, C. Baines, and S. Takagi, Phys. Rev. Lett. 96, 247203 (2006).

${ }^{28}$ A. Yaouanc and P. Dalmas de Réotier, Muon Spin Rotation, Relaxation, and Resonance; Applications to Condensed Matter (Oxford University Press, Oxford, UK, 2011), p. 271.

${ }^{29}$ A. Keren, J. S. Gardner, G. Ehlers, A. Fukaya, E. Segal, and Y. J. Uemura, Phys. Rev. Lett. 92, 107204 (2004).

${ }^{30}$ J. S. Helton, K. Matan, M. P. Shores, E. A. Nytko, B. M. Bartlett, Y. Qiu, D. G. Nocera, and Y. S. Lee, Phys. Rev. Lett. 104, 147201 (2010).

${ }^{31}$ C.-Y. Liu, D. E. MacLaughlin, A. H. Castro Neto, H. G. Lukefahr, J. D. Thompson, J. L. Sarrao, and Z. Fisk, Phys. Rev. B 61, 432 (2000). 